\title{
Intercostal ancient schwannoma mimicking an apical lung tumour
}

A 56-year-old woman was admitted to our hospital with inspiratory dyspnoea, dry cough, vague right chest pain and right arm pain for 2 months. She denied tobacco use or asbestos exposure and had no significant medical or family history.

A chest radiograph highlighted extensive radiopacity involving the superior right lobe, the lateral view detailed the mass as being located in the posterior mediastinum. Chest CT scanning revealed a large well circumscribed heterogeneous tumour of $7 \times 6 \times 5 \mathrm{~cm}$, with punctuate calcifications and focal low density areas, located in the posterior mediastinum in the right paravertebral gutter (fig 1). MRI demonstrated a well defined lobulated mass and inhomogeneous enhancement with non-enhanced linear and patchy areas after intravenous gadolinium injection. There were no expansions through spinal roots or other tissues (fig 2). The sample taken by CT guided fine needle aspiration of the mass was of equivocal

\section{Learning points}

- Ancient schwannoma is a rare variant of a neurilemoma.

- The intercostal ancient schwannoma can mimic pulmonary neoplasm in chest radiographs and $\mathrm{CT}$.

- Ancient schwannoma shows degenerative histological changes which may lead to a mistaken diagnosis of malignant neoplasm.

value because it showed atypical features. The diagnosis of malignant tumour was assumed, and the patient underwent surgical exploration to determine the final histological diagnosis. The tumour, being of extrapleural origin, arising from intercostal nerve and having no intracanalicular extension, was isolated with a complete en bloc surgical resection. The histological diagnosis was "ancient schwannoma" (fig 3). The postoperative course was uneventful and the patient was discharged home on postoperative day 6.

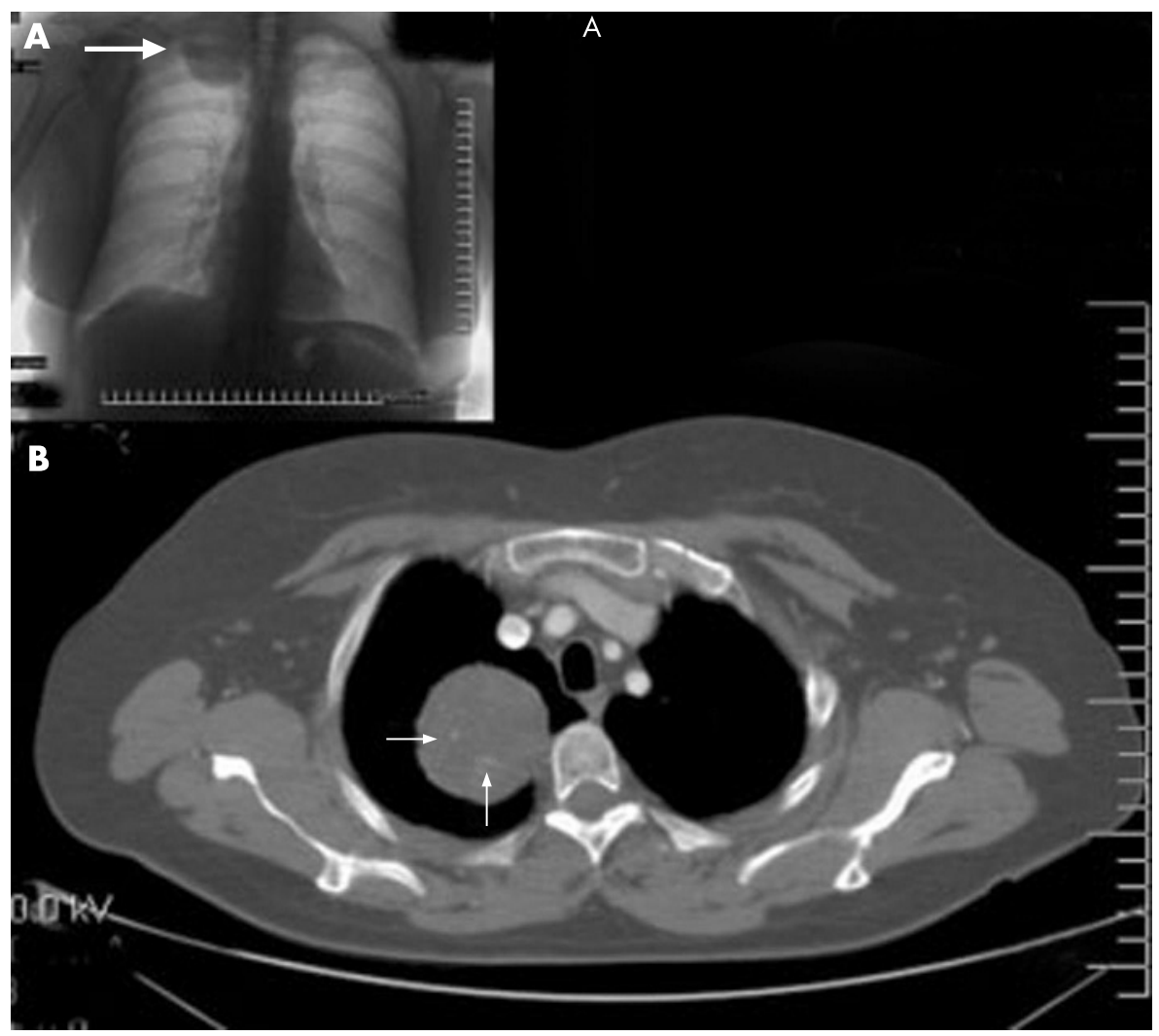

Figure 1 (A) Chest radiograph showed a large neoplasm of the apex lung (arrow). (B) Chest CT scan revealed a well delineated heterogeneous mass with punctuate calcifications (arrows) of the right paravertebral gutter.

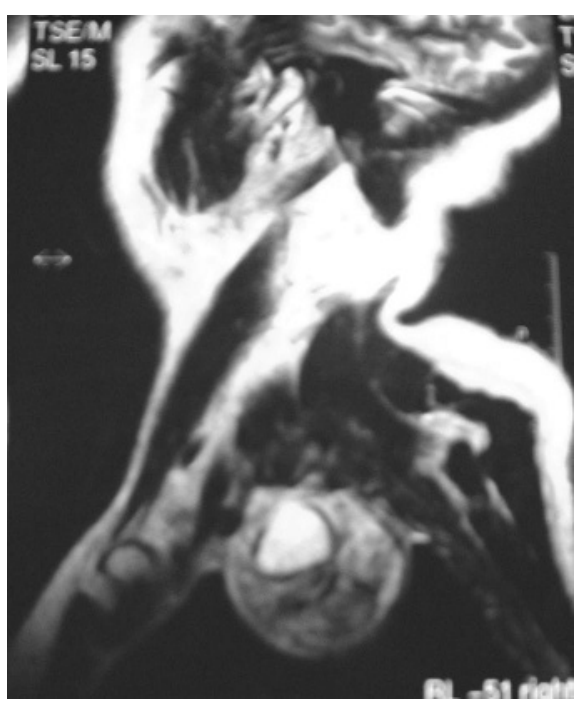

Figure 2 Chest MRI, after intravenous injection of contrast, showed an inhomogeneous mass with non-enhanced linear and patchy areas, representing fibrous septal structures and areas of cystic degeneration.

The intercostal ancient schwannoma can mimic pulmonary neoplasm in chest radiographs and $\mathrm{CT} .{ }^{1} \mathrm{MRI}$ is the technique of choice in the assessment of peripheral nerve tumours. ${ }^{2}$ Ancient schwannoma is a rare variant of a neurilemoma and shows degenerative histological changes which may lead to a mistaken diagnosis of malignant neoplasm. The definitive diagnosis is possible only after histopathological examination.

\section{F Petteruti, ${ }^{1}$ G De Luca, ${ }^{2}$ A Lerro, ${ }^{1}$ A Luciano, ${ }^{1}$ I Cozzolino, ${ }^{3}$ P Pepino ${ }^{1}$}

${ }^{1}$ Cardiothoracic Surgery Units, "Pineta Grande" Clinic, Castel Volturno, Caserta, Italy; ${ }^{2}$ Thoracic Surgery Units, University Federico II, Naples, Italy; ${ }^{3}$ Dipartimento di Scienze Biomorfologiche e Funzionali, Servizio di Anatomia Patologica, University Federico II, Naples, Italy 

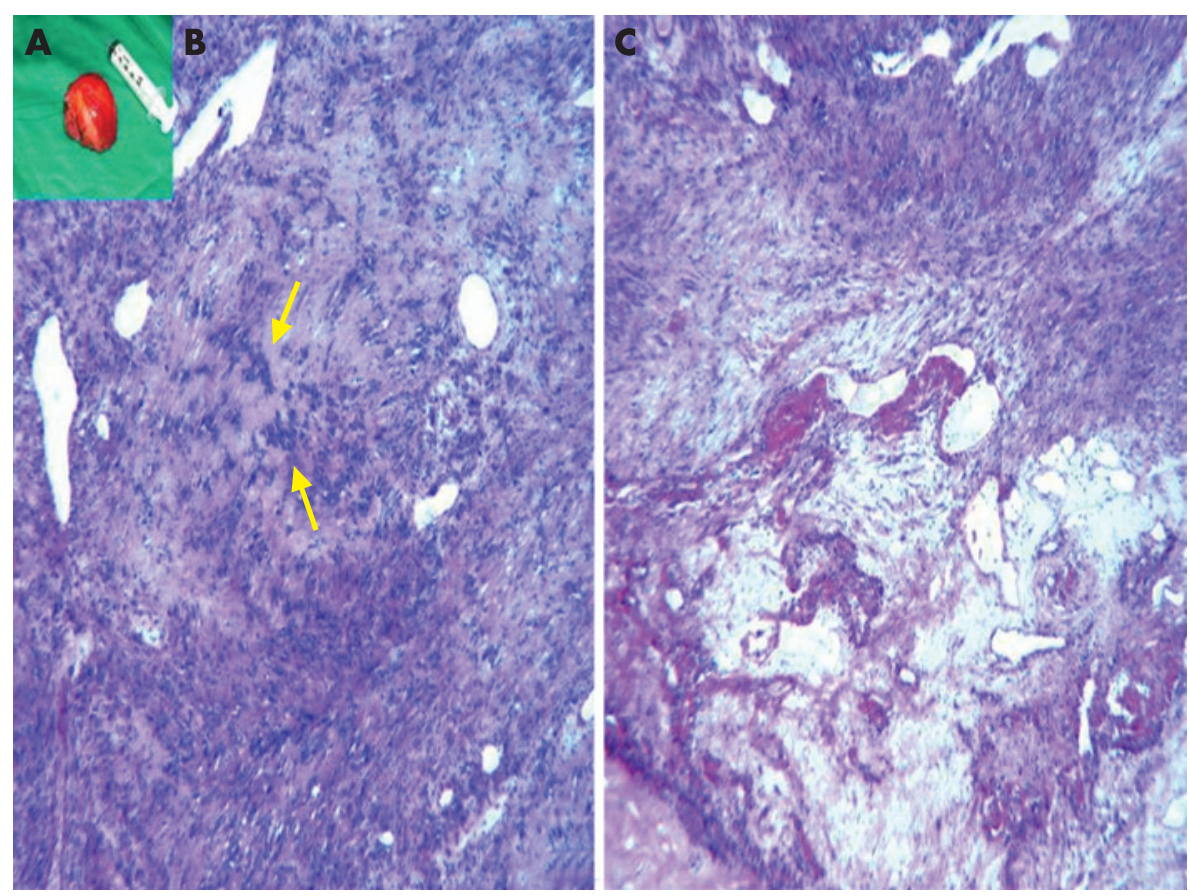

Correspondence to: Dr A Lerro, Via Diocleziano, 178 Napoli, Italy; cardiothorax.pineta@libero.it

Accepted 2 July 2007

Competing interests: None.

Patient consent: Obtained

Thorax 2008:63:845-846. doi:10.1136/thx.2007.082495

\section{REFERENCES}

1. Rodriguez CA, Munhoz AH, Zampier JA, et al. Benign intercostal nerve schwannoma simulating pulmonary neoplasm: case report. Arq Neuropsiquiatr 2004:62:1100-3

2. Isobe K, Shimizu T, Akahane T, et al. Imaging of ancient schwannoma. AJR Am J Roentgenol 2004;183:331-6.

Figure 3 (A) At gross examination the mass measured $7 \times 6 \times 5 \mathrm{~cm}$, appeared firm, encapsulated, smoothly lobulated and well circumscribed. (B) Hypercellular area where characteristically the nuclei and cytoplasm line up with adjacent cells with formation of so-called Verocay bodies (yellow arrows) (haematoxylin-eosin, original magnification $\times 10$ ). (C) Thick walled vessels with prominent hyalinisation (haematoxylin-eosin, original magnification $\times 20$ ). 